\title{
Dynamics of orientational ordering in fluid membranes
}

\author{
Nariya Uchidat \\ Yukawa Institute for Theoretical Physics, Kyoto University, Kyoto 606-8502, Japan
}

(Submitted February 28, 2002; revised July 31, 2002)

\begin{abstract}
We study the dynamics of orientational phase ordering in fluid membranes. Through numerical simulation we find an unusually slow coarsening of topological texture, which is limited by subdiffusive propagation of membrane curvature. The growth of the orientational correlation length $\xi$ obeys a power law $\xi \propto t^{w}$ with $w<1 / 4$ in the late stage. We also discuss defect profiles and correlation patterns in terms of long-range interaction mediated by curvature elasticity.

87.16.Dg, 64.70.Md, 64.60.Cn, 05.70.Fh
\end{abstract}

Fluid membranes exhibit a variety of shape transformations due to their flexibility and sensitivity to changes in temperature and osmotic pressure. They are most simply modeled by a structureless deformable surface with bending energy and a few constraints on its global geometry [1]. More diverse and prominent deformations occur in membranes with internal degrees of freedom, especially when they undergo a transition between different thermodynamic phases. An example is provided by a multicomponent membrane in two-phase coexistence, deformation of which is controlled by a local coupling between composition and curvature. The equilibrium morphology as well as the dynamics of phase separation have been addressed in a number of theoretical studies $[2,3,4,1,5,6,67,8,8,97$, which have relevance to the so-called budding and other experimental observations.

Another class of shape transformations is characterized by a coupling to an orientational degree of freedom. It represents anisotropic in-plane shape and/or configuration of the constituent molecules. The possibility of quasi-long-range orientational order has been proposed [10, 11, 12] and the resulting morphologies (such as tubules, sponges, and egg-cartons) and defect energetics have been explored 11, 12, 13, 14, 15, 16, 17]. A special case of this is the nematic order [12,13,14, 15, 16], the presence of which is suggested 12] in the so-called "gemini" surfactants (each of which is a pair of molecules covalently linked at their polar heads) [18]. It is recently shown that the nematic order, in combination with chirality, accounts for the formation of helical ribbons by the material [16].

In this paper, we address the nematic phase ordering from a dynamical perspective, which has been lacking so far. Via numerical simulation, we show that the coarsening of topological texture is greatly decelerated by the coupling to membrane deformation. The growth of texture size is asymptotically characterized by a power law with an exponent less than $1 / 4$, instead of the $1 / 2$ for ordinary nematic liquid crystals 19,20.21. We provide the interpretation of this novel non-equilibrium effect. To prepare for this, we also study how the curvature-orientation coupling controls defects and correlation structure in mechanical equilibrium. Our results follow from a Ginzburg-Landau model in the simplest setting of a flat topology under a weak coupling condi- tion. It would be straightforward to include more complicated though realistic aspects, such as the stiffness anisotropy 10, 16], non-flat topologies [13], and interlayer coupling [14.15].

We consider an initially flat membrane without any overhang, and describe its profile in the Monge gauge as $z=h(\boldsymbol{r})=h(x, y)$. The orientational order parameter $Q_{i j}(\boldsymbol{r})=Q_{i j}(x, y)$ is a symmetric traceless tensor related to the scalar order parameter $S$ and nematic director $n_{i}$ as $Q_{i j}=S\left(n_{i} n_{j}-\delta_{i j} / 2\right)(i, j=x, y)$. The model free energy consists of three parts, which are the homogeneous, Frank, and curvature-elastic contributions. Under the assumption $|\nabla h| \ll 1$, we retain the lowest order terms with respect to $\nabla h$, as

$$
\begin{aligned}
F & =\int d \boldsymbol{r}\left(f_{\text {hom }}+f_{F}+f_{\text {curv }}\right), \\
f_{\text {hom }} & =\frac{A}{2} Q_{i j}^{2}+\frac{C}{4}\left(Q_{i j}^{2}\right)^{2}, \\
f_{F} & =\frac{M}{2}\left(\partial_{i} Q_{j k}\right)^{2}, \\
f_{\text {curv }} & =\frac{\kappa}{2}\left(\nabla^{2} h\right)^{2}+\alpha E_{i j} Q_{j k} \partial_{i} \partial_{k} h
\end{aligned}
$$

(summation over repeated indices is implied). Here, $A$, $C$, and $M$ are the Landau-de Gennes coefficients [22], $\kappa$ is the bending rigidity, and $\alpha$ is the coupling constant. Finally, $E_{i j}$ is the unit tensor for an non-chiral nematic membrane 12 and the totally antisymmetric tensor for a chiral nematic [16]. From now on we concentrate on nonchiral membranes, but discussions are parallel for chiral ones, as we shall see. Neglecting long-range hydrodynamic interactions mediated by the solvent, we write the kinetic equations in the form

$$
\frac{\partial Q_{i j}}{\partial t}=-\Gamma_{Q}\left(\frac{\delta F}{\delta Q_{i j}}\right)^{(s)}
$$

where $(s)$ denotes the symmetric traceless part, and

$$
\frac{\partial h}{\partial t}=-\Gamma_{h} \frac{\delta F}{\delta h}=-\Gamma_{h}\left(\kappa \nabla^{2} \nabla^{2} h+\alpha \partial_{i} \partial_{j} Q_{i j}\right) .
$$

To analyze the initial growth of order parameter upon a quench into the nematic phase, we linearize Eqs.(5) with the aid of the unitary transformation

${ }^{*}$ Present address: Department of Physics, Tohoku University, 980-8578, Japan. 


$$
\begin{aligned}
{\left[\begin{array}{l}
Q_{+}(\boldsymbol{q}) \\
Q_{-}(\boldsymbol{q})
\end{array}\right] } & =\left[\begin{array}{rr}
\cos 2 \zeta & \sin 2 \zeta \\
-\sin 2 \zeta & \cos 2 \zeta
\end{array}\right]\left[\begin{array}{l}
Q_{x x}(\boldsymbol{q}) \\
Q_{x y}(\boldsymbol{q})
\end{array}\right] \\
\zeta & =\arctan \left(q_{y} / q_{x}\right) .
\end{aligned}
$$

The three eigenmodes are expressed as

$$
e_{ \pm}(\boldsymbol{q})=Q_{+}(\boldsymbol{q})+\frac{1}{2}\left[c(q) \pm \sqrt{c(q)^{2}+\frac{4 \Gamma_{Q}}{\Gamma_{h}}}\right] h(\boldsymbol{q})
$$

and $e_{0}(\boldsymbol{q})=Q_{-}(\boldsymbol{q})$, where $c(q)=-\kappa q^{2} / \alpha+\Gamma_{Q}(A+$ $\left.M q^{2}\right) /\left(\Gamma_{h} \alpha q^{2}\right)$. Their growth rates $\gamma_{a}=\partial\left(\ln e_{a}\right) / \partial t$ $(a=+,-, 0)$ read

$$
\begin{array}{r}
\gamma_{ \pm}(q)=\frac{1}{2}\left[-\Gamma_{Q}\left(A+M q^{2}\right)-\Gamma_{h} \kappa q^{4}\right. \\
\left. \pm \Gamma_{h} \alpha q^{2} \sqrt{c(q)^{2}+\frac{4 \Gamma_{Q}}{\Gamma_{h}}}\right],
\end{array}
$$

and $\gamma_{0}(q)=-\Gamma_{Q}\left(A+M q^{2}\right)$. The spinodal point is located at $A=\alpha^{2} / \kappa$ 12, 14 as seen from the behavior of $\gamma_{a}(q)$ at small $q$.

The nonlinear dynamics of phase ordering is studied by numerically integrating Eqs. (5.6) on a square lattice. We choose $A=-1, C=20, M=1, \kappa=20$, and $\alpha=1$ as the standard parameter set, with the mesh size $\Delta x=1$. The Landau coefficients give the equilibrium scalar order parameter $S_{e q} \sim \sqrt{2|A| / C} \simeq 0.3$ and the defect core size $l_{\text {core }} \sim \sqrt{M /|A|}=1$. The large ratio $\kappa / M=20$ is not unrealistic if we consider the large stiffness $\kappa \sim 20 k_{B} T$ usually found in biological membranes. We impose periodic boundary conditions on a $512 \times 512$ lattice. For the initial condition, random numbers uniformly distributed in $[-0.1,0.1]$ are assigned to each component of $Q_{i j}$. The kinetic equations are integrated using the Euler scheme with $\Gamma_{Q}=0.1, \Gamma_{h}=0.1$ and the step increment $\Delta t=0.025$. Note that the flat membrane approximation breaks down as the texture size exceeds the equilibrium curvature radius $\sim \kappa /\left(\alpha S_{e q}\right)$. All the data shown below are taken from the time region in which $\langle|\nabla h|\rangle<0.15$ (we denote a spatial average by $\langle\cdots\rangle$ ).

Shown in Fig.1 are snapshots of the Schlieren texture $Q_{x y}^{2}(\boldsymbol{r})$ and the mean curvature $\nabla^{2} h(\boldsymbol{r})$. In the late stage, the coarsening proceeds via pair annihilation of topological defects with $s=+1 / 2$ and $-1 / 2$, where $s$ is the winding number for the apolar vector $\boldsymbol{n}$ (or the disclination strength [23]). It is seen that a $-1 / 2$ defect accompanies three lobes of positive mean curvature, while $\mathrm{a}+1 / 2$ defect has one. Where the director points to the defect core, $\nabla^{2} h$ is negative (positive) for a $+1 / 2$ $(-1 / 2)$ defect (resp.). To understand this, we note that, the membrane shape in the latest stage of coarsening is locally well equilibrated with respect to the order parameter configuration. Then, for analysis of static correlation properties, we can assume the mechanical equilibrium condition $\delta F / \delta h=0$, or

$$
\nabla^{2}\left(\nabla^{2} h\right)=-\frac{\alpha}{\kappa} \partial_{i} \partial_{j} Q_{i j}
$$

This can be solved in the Fourier space as $h(\boldsymbol{q})=$ $\left(\alpha / \kappa q^{2}\right) Q_{+}(\boldsymbol{q})$, substitution of which into (雨) gives the effective curvature elastic energy as

$$
F_{\text {curv }}^{(e f f)}=-\frac{\alpha^{2}}{2 \kappa} \int \frac{d \boldsymbol{q}}{(2 \pi)^{2}}\left|Q_{+}(\boldsymbol{q})\right|^{2} .
$$

It becomes dominant over Frank elasticity when the texture size is larger than the characteristic length,

$$
\lambda=\sqrt{\kappa M} / \alpha
$$

Within this distance from a defect, the optimum configuration $Q_{i j}(\boldsymbol{r})$ is little affected by the elastic coupling and approximately minimizes Frank elastic energy. By further assuming $S=$ const., $\boldsymbol{n}=(\cos \theta, \sin \theta)$, and the polar coordinate system $(r, \phi)$, the approximation gives 23]

$$
\theta=s\left(\phi-\phi_{0}\right), \quad \phi_{0}=\text { const. }
$$

for a defect of index $s$ located at the origin. The Poisson equation (10) for mean curvature has the special solution,

$$
\frac{\kappa}{\alpha S} \nabla^{2} h= \begin{cases}\frac{s}{2 s-2} \cos [2(\theta-\phi)] & (s \neq 1), \\ 0 & (s=1) .\end{cases}
$$

This qualitatively explains the mean curvature profile obtained by simulation. It also shows that a $+1 / 2$ defect carries much larger bending energy than its anti-defect. On the other hand, the anisotropic part of the curvature

$$
H_{i j}=\partial_{i} \partial_{j} h-\left(\nabla^{2} h / 2\right) \delta_{i j}
$$

is proportional to $Q_{i j}$ in a uniform equilibrated system, and the principal curvature axis is parallel to the director. This holds also in the coarsening system in the late stage and except in the vicinity of defects, as we confirmed numerically.

The long-range elastic interaction also modifies orientational correlation at lengthscales larger than $\lambda$. In the real space, and under the approximation $S=$ const., Eq.(11) is rewritten as

$$
F_{\text {curv }}^{(e f f)}=-\frac{\alpha^{2} S^{2}}{16 \pi \kappa} \iint d \boldsymbol{r} d \boldsymbol{r}^{\prime} \frac{\cos 2\left[\theta_{r e l}\left(\boldsymbol{r}, \boldsymbol{r}^{\prime}\right)+\theta_{r e l}\left(\boldsymbol{r}^{\prime}, \boldsymbol{r}\right)\right]}{\left|\boldsymbol{r}-\boldsymbol{r}^{\prime}\right|^{2}},
$$

where $\theta_{\text {rel }}\left(\boldsymbol{r}, \boldsymbol{r}^{\prime}\right)$ is the angle between $\boldsymbol{n}(\boldsymbol{r})$ and $\boldsymbol{r}^{\prime}-\boldsymbol{r}$ [27]. It suggests that orientational correlation is enhanced in directions parallel and perpendicular to the local director, while it is suppressed in oblique directions. This can be quantified by the relative orientation correlation function, which we define as

$$
G_{r e l}\left(\boldsymbol{r}-\boldsymbol{r}^{\prime}\right)=\left\langle Q_{i j}(\boldsymbol{r}) Q_{i j}\left(\boldsymbol{r}+U(\boldsymbol{n}(\boldsymbol{r})) \cdot\left(\boldsymbol{r}^{\prime}-\boldsymbol{r}\right)\right)\right\rangle
$$

where $U(\boldsymbol{n}(\boldsymbol{r}))$ is a matrix of rotation that maps $\boldsymbol{n}(\boldsymbol{r})$ onto $\boldsymbol{e}_{x}$. Simulation result in Fig.2 shows that the expected tendency is prominent in the region $\left|\boldsymbol{r}-\boldsymbol{r}^{\prime}\right| \gg \lambda$, where correlation in oblique directions is negative.

The coarsening kinetics is monitored through the orientational correlation length $\xi_{Q}$, which is defined by the correlation function $G_{Q}\left(\left|\boldsymbol{r}-\boldsymbol{r}^{\prime}\right|\right)=\left\langle Q_{i j}(\boldsymbol{r}) Q_{i j}\left(\boldsymbol{r}^{\prime}\right)\right\rangle$ via $G_{Q}\left(\xi_{Q}\right) / G_{Q}(0)=1 / 2$. Similarly, the curvature correlation length $\xi_{H}$ is defined as the half-value decay length of $G_{H}\left(\left|\boldsymbol{r}-\boldsymbol{r}^{\prime}\right|\right)=\left\langle H_{i j}(\boldsymbol{r}) H_{i j}\left(\boldsymbol{r}^{\prime}\right)\right\rangle$. In Fig.3, the orientational correlation length is plotted as a function of time. In the absence of coupling, $\xi_{Q}$ is well fitted by a power law $\xi_{Q} \propto t^{w_{0}}$ with $w_{0}=0.43 \pm 0.02$, in good agreement with previous results [21,24]. Remarkably, the 
elastic coupling makes the growth much slower. The instantaneous growth exponent

$$
w_{Q}(t)=d\left(\ln \xi_{Q}\right) / d(\ln t)
$$

decreases from an initial value $\simeq w_{0}$, and then converges to a much smaller value $w_{\infty}$ as $t \rightarrow \infty$. Deviation from the initial value starts earlier for a stronger coupling. In the intermediate stage, $w_{Q}(t)$ shows an undershoot and even turns negative for most of the parameters we studied. The asymptotic exponent $w_{\infty}$ is estimated to be $0.12 \pm 0.02$ for $\alpha=2$ and $0.13 \pm 0.01$ for $\alpha=3$ [28]. On the other hand, the curvature coarsening exponent $w_{H}(t)=d\left(\ln \xi_{H}\right) / d(\ln t)$ first decreases monotonically and then converges to the same value $w_{\infty}$ within error bars. In the bottom of the figure we plot the ratio $\xi_{Q} / \xi_{H}$, which decays to unity after an initial increase. In the same figure, we indicate the time at which the ratio $\left\langle\left|f_{\text {curv }}\right|\right\rangle /\left\langle f_{F}\right\rangle$ reaches 2 . It shows that the two lengths merge as the curvature-orientational coupling becomes dominant over Frank elasticity.

How does the coupling decelerate coarsening? First we note that the effective elastic interaction (11) does not provide an explanation. In the mechanical equilibrium, the free energy density $f_{\text {curv }}$ is reduced to its homogeneous minimum except in a region of size $\sim \lambda$ around each defect, in which Frank and curvature elastic energies are balanced. Thus the $\xi_{Q}$-dependence of $f_{\text {curv }}$ is that of the defect density $\propto 1 / \xi_{Q}^{2}$, which is also the scaling of Frank elastic energy (except for a logarithmic correction). Therefore, the effective elastic interaction cannot affect the coarsening exponent. This was confirmed by an additional simulation that directly implements the longrange interaction using Fourier transformation. It shows that (i) coarsening curves $\xi_{Q}(t)$ and $\xi_{H}(t)$ are both well fitted by the exponent 0.43 for null coupling; (ii) dissipation rates $d\left\langle f_{F}\right\rangle / d t$ and $d\left\langle f_{\text {curv }}\right\rangle / d t$ are of the same order and their ratio is roughly constant in the late stage.

Thus we can attribute the origin of slow coarsening to the dynamics of shape change, Eq.(6). It is subdiffusive and the source term $\left(\propto \partial_{i} \partial_{j} Q_{i j}\right)$ is small except near defects. According to a simple dimensional counting, the mean defect separation scales like $\xi_{Q}$ and the timescale of curvature relaxation is proportional to $\xi_{Q}^{4}$. If $w_{\infty}>1 / 4$, the coarsening of curvature field cannot keep up with that of order parameter, and hence $f_{\text {curv }}$ increases. This is energetically disfavored once the coupling becomes dominant over Frank elasticity. Therefore, the asymptotic exponent cannot exceed $1 / 4$. The reason why $w_{\infty}$ is even smaller than $1 / 4$ is not very simple. A possible explanation is the logarithmic correction to the $\xi_{Q}$-dependence of Frank elastic energy. In the XY model and ordinary nematic fluids in $2 \mathrm{D}$, it causes a logarithmic correction to the power law [29] and the apparent exponent is smaller than $1 / 2$ in finite time simulations $[20,21,24,25,26]$. A similar effect is expected for the present case, because the coarsening is still driven by Frank elasticity.

The non-monotonicity of $\xi_{Q}(t)$ in the intermediate stage corresponds to the fact that Frank elastic energy is temporally sacrificed to reduce curvature elastic energy, which has initially grown on the smaller scale $\xi_{H}$. Because $\xi_{Q}$ grows faster than $\xi_{H}$ in the initial stage, it is at least necessary that $w_{Q}<w_{H}$ holds temporally for the two lengths to finally merge.
Some remarks are in order. First, hydrodynamic flow of the solvent effectively modifies the mobility as $\Gamma_{h}(q)=1 /(4 \eta q)(\eta$ : viscosity $)$ [1]. However, the curvature dynamics is still subdiffusive, and the coarsening exponent cannot exceed $1 / 3$. We have performed a simulation incorporating the hydrodynamic interaction, by solving Eq.(6) with $\Gamma_{h}(q)$ above in the Fourier space and transforming back each time step to solve Eq.(5) in the real space. We found the coarsening exponent $\xi_{Q}$ to be $0.17 \pm 0.03$ (for $\alpha=2$ and $\eta=1.25$ ), which confirms the weakness of hydrodynamic accerelation. The details of this simulation will be presented elsewhere.

Second, the mechanism of slow coarsening is unique to membranes with a continuous degree of freedom and is irrelevant to phase separation dynamics, which is described by a scalar order parameter [6, [, 8]. This has the following reason. In phase separation, significant changes in the order parameter occur only at domain boundaries. However, domain boundaries are also the sources of curvature propagation, as we can see from the corresponding dynamic equation for $h$. Therefore, the membrane shape can immediately follow the composition change. In contrast, the orientational order parameter changes significantly even far from moving defects. In order to adapt to this, shape deformations spread from the defects and over the whole texture, which is slow.

Finally we consider the effect of chirality. With the antisymmetric coupling between curvature and $Q_{i j}$, a straightforward calculation gives the elastic interaction as

$$
F_{\text {curv }}^{(e f f)}=-\frac{\alpha^{2}}{2 \kappa} \int \frac{d \boldsymbol{q}}{(2 \pi)^{2}}\left|Q_{-}(\boldsymbol{q})\right|^{2} .
$$

The chirality reverses the correlation anisotropy so that the director correlation is enhanced in oblique directions. However, the coarsening law is unaltered since the last term in the membrane's dynamic equation (6) is still proportional to $\nabla \nabla Q$ and sources of curvature propagation are localized around defects.

This work is supported by the Grant in Aid for Scientific Research from Japan Society for the Promotion of Science.

[1] For a review, see: U. Seifert, Adv. Phys. 46, 13 (1997).

[2] S. Leibler, J. Phys. (France) 47, 507 (1986).

[3] D. Andelman et al., Europhys. Lett. 19, 57 (1992).

[4] F. Jülicher and R. Lipowsky, Phys. Rev. Lett. 70, 2964 (1993).

[5] U. Seifert, Phys. Rev. Lett. 70, 1335 (1993).

[6] T. Taniguchi, Phys. Rev. Lett. 76, 4444 (1996).

[7] P. B. Sunil Kumar and M. Rao, Phys. Rev. Lett. 80, 2489 (1998).

[8] P. B. Sunil Kumar et al., Phys. Rev. Lett. 86, 3911 (2001).

[9] Y. Jiang et al., Phys. Rev. E 61, R57 (2000).

[10] W. Helfrich and J. Prost, Phys. Rev. A 38, 3065 (1988).

[11] F. C. MacKintosh and T. C. Lubensky, Phys. Rev. Lett. 67, 1169 (1991).

[12] J. B. Fournier and P. Galatola, J. Phys. II France 7, 1509 (1997). 
[13] T. C. Lubensky and J. Prost, J. Phys. II France 2, 371 (1992).

[14] U. Seifert et al., Phys. Rev. Lett. 77, 5237 (1996).

[15] J. -B. Fournier and L. Peliti, Phys. Rev. E 58, R6919 (1998).

[16] R. Oda et al., Nature 399, 566 (1999).

[17] J. V. Selinger et al., Phys. Rev. E 53, 3804 (1996).

[18] R. Zana and Y. Talmon, Nature 362, 228 (1993).

[19] I. Chuang et al, Science 251, 1336 (1991).

[20] H. Toyoki, Phys. Rev. E 47, 2558 (1993).

[21] M. Zapotocky et al., Phys. Rev. E 51, 1216 (1995).

[22] The 3rd order term $\propto Q_{i j} Q_{j k} Q_{k i}$ in the Landau expansion identically vanishes for the $2 \mathrm{D}$ traceless symmetric tensor $Q_{i j}$.

[23] S. Chandrasekhar, Liquid Crystals (Cambridge Univ. Press, Cambridge, 2nd Ed. 1992).

[24] R. E. Blundell and A. J. Bray, Phys. Rev. E 49, 4925 (1994).

[25] M. Mondello and N. Goldenfeld, Phys. Rev. A 42, 5865 (1990).

[26] B. Yurke et al., Phys. Rev. E 47, 1525 (1993).

[27] A similar expression has been obtained for interaction between anisotropic membrane inclusions: J. -M. Park and T. C. Lubensky, J. Phys. I France 6, 1217 (1996).

[28] This estimate uses least-square-fits in the time region where $\xi_{Q}$ and $\xi_{H}$ differ by less than $5 \%$. The data for $\alpha=2$ are taken from long runs up to $t=204800$.

[29] A. J. Bray, Adv. Phys. 43, 357 (1994).

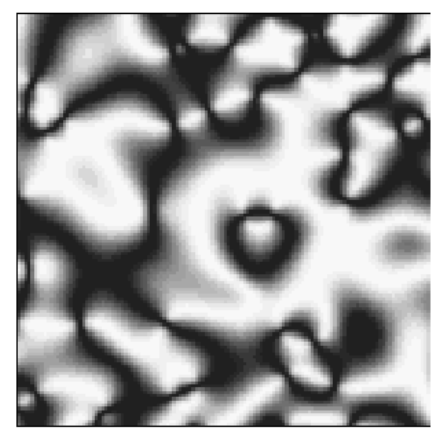

$\mathrm{t}=400$

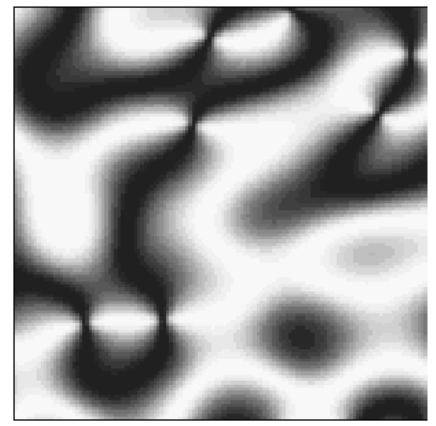

$t=6400$

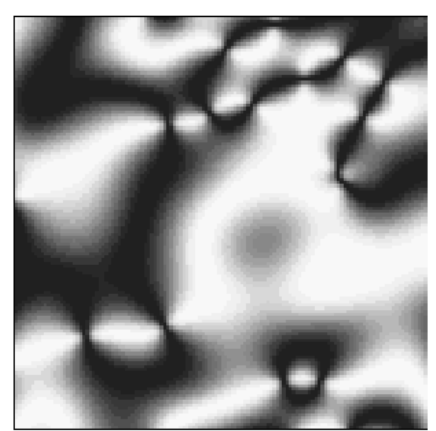

$t=1600$

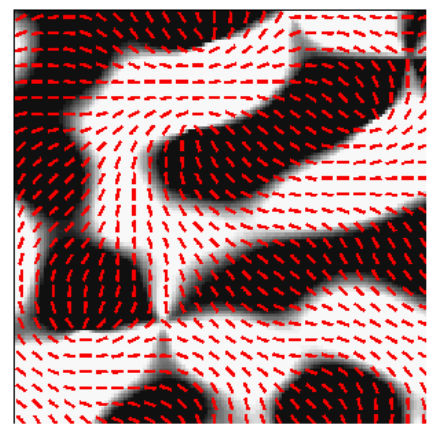

$\mathrm{t}=6400$ (curvature)
FIG. 1. Snapshots of $Q_{x y}^{2}(\mathbf{r})$ on a $128^{2}$ portion of the lattice, at $t=400,1600$, and 6400 . The last panel shows the mean curvature $\nabla^{2} h(\mathbf{r})$ with the director field in short lines.

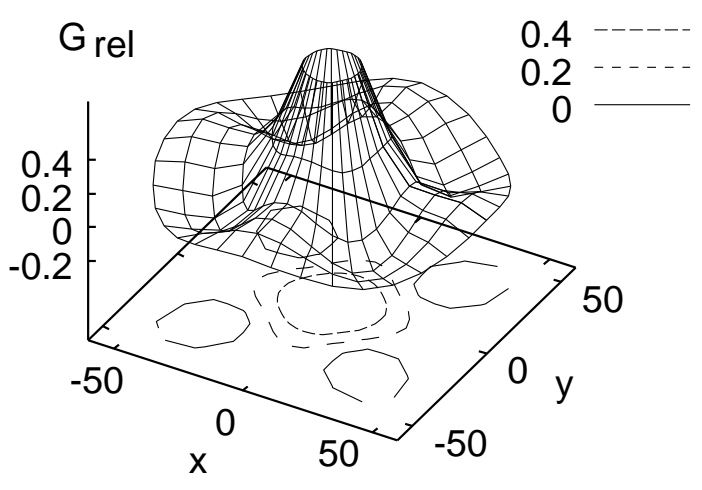

FIG. 2. Relative orientation correlation function $G_{r e l}(\mathbf{r})$ at $t=6400$ (averaged over 10 independent runs).

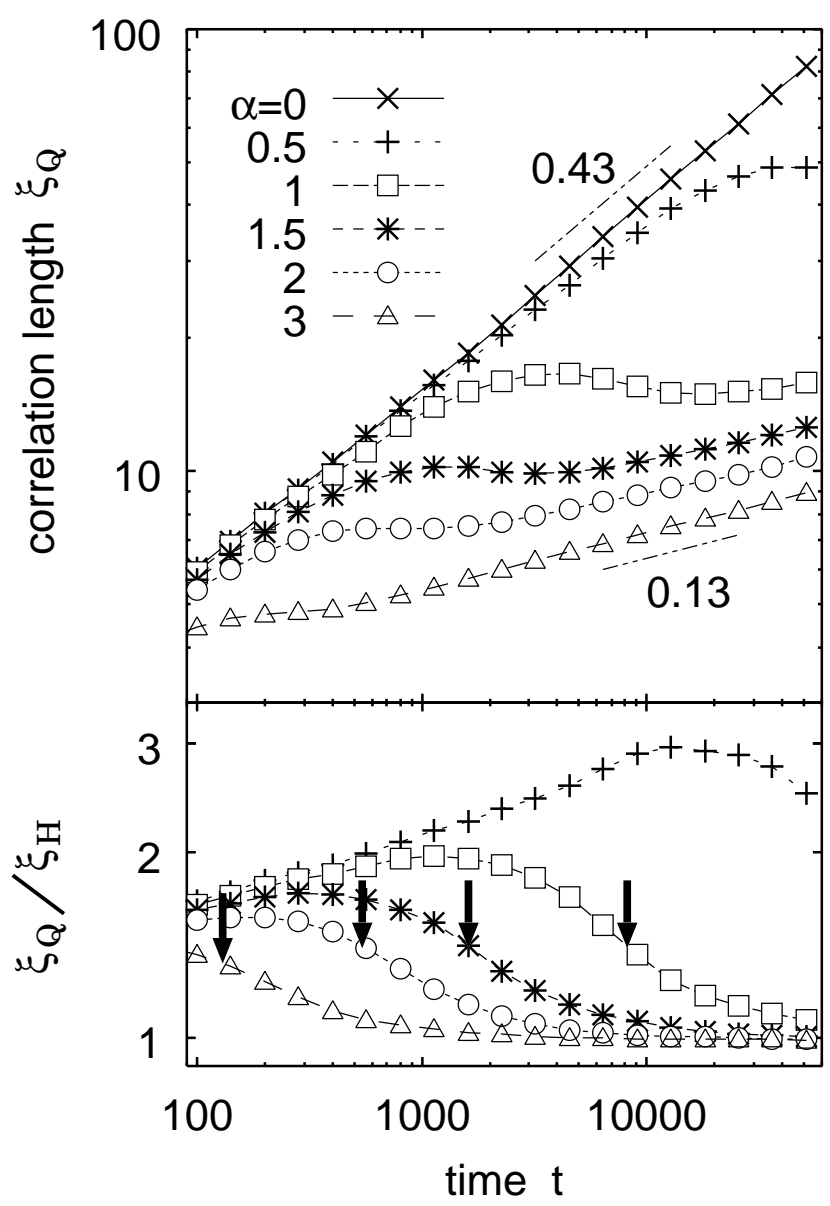

FIG. 3. Top: orientational correlation length $\xi_{Q}$ versus time. The lines corresponding to $w_{Q}=0.13$ and 0.43 are drawn as guides to the eye. Bottom: the ratio between orientational and curvature correlation lengths. Arrows indicate the time at which $\left\langle\left|f_{\text {curv }}\right|\right\rangle /\left\langle f_{F}\right\rangle=2$. 